\title{
Estimulação elétrica neuromuscular na força muscular e no desempenho motor de
}

\section{idosos: uma revisão sistemática}

Neuromuscular electrical stimulation in muscle strength and motor performance of the elderly: a

\author{
systematic review
}

Estimulación eléctrica neuromuscular en la fuerza muscular y en desempeño motor de gerontes: un repaso sistemático

\author{
Mariana Correia Figueira \\ ORCID: https://orcid.org/0000-0002-6186-7009 \\ Faculdade Independente do Nordeste, Brasil \\ E-mail: figueiracmariana@gmail.com \\ Ana Clara Nunes Oliveira Ferreira \\ ORCID: https://orcid.org/0000-0002-2873-2487 \\ Faculdade Independente do Nordeste, Brasil \\ E-mail: anaclaranunes98@outlook.com \\ Lucas Silveira Sampaio \\ ORCID: https://orcid.org/0000-0003-4246-7947 \\ Faculdade Independente do Nordeste, Brasil \\ E-mail: lucassilveira@fainor.com.br
}

\begin{abstract}
Resumo
Este estudo teve o objetivo de avaliar a literatura científica de forma sistemática para analisar o efeito da EENM no ganho de força muscular e no desempenho motor em idosos. O levantamento bibliográfico foi realizado através de publicações indexadas na base de dados da Biblioteca Virtual de Saúde, Scientific Electronic Library Online, Medical Literature Analysis and Retrieval System Online, US National Library of Medicine National Institutes of Heath e Physiotherapy evidence database. A busca foi realizada utilizando os descritores na língua portuguesa e inglesa: "Terapia por estimulação elétrica", "Força muscular", "Desempenho Motor", "Idoso". Para organização do estudo, foram seguidos os critérios da revisão sistemática PRISMA (Principais Itens para Relatar Revisões Sistemáticas e Metanálises). Após avaliação, foram selecionados cinco artigos que se enquadravam nos critérios de inclusão. Diversos estudos mostraram dados que fornecem evidências de que a eletroestimulação neuromuscular é um método seguro para combater o declínio muscular em idosos, como também evita a perda da força muscular e proporciona seu incremento. Esta revisão foi conclusiva no tocante à eficácia da EENM, pois demonstrou que há efetivamente aumento da força de MMII e melhora no desempenho motor após um protocolo de EENM em idosos, mesmo com grande divergência nos parâmetros e no tempo de intervenção.
\end{abstract}

Palavras-chave: Terapia por estimulação elétrica; Força muscular; Desempenho motor; Idoso.

\begin{abstract}
This study aimed to evaluate the scientific literature in a systematic way to analyze the effect of NMES on muscle strength gain and motor performance in the elderly. The bibliographic survey was carried out through publications indexed in the database of the Virtual Health Library, Scientific Electronic Library Online, Medical Literature Analysis and Retrieval System Online, US National Library of Medicine National Institutes of Health and Physiotherapy evidence database. The search was carried out using the descriptors in Portuguese and English: "Electrical Stimulation Therapy", "muscle strength", "motor performance" and "elderly". To organize the study, the criteria of the PRISMA systematic review (Preferred Reporting Items for Systematic Reviews and Meta-Analyses) were followed. After evaluation, five articles were selected that met the inclusion criteria. Several studies have shown data that provide evidence that neuromuscular electrostimulation is a safe method to combat muscle decline in the elderly, as well as prevents the loss of muscle strength and provides its increase. This review was conclusive regarding the effectiveness of NMES, as it demonstrated that there is effectively an increase in the strength of MMII and improvement in motor performance after an NMES protocol in the elderly, even with great divergence in parameters and intervention time.
\end{abstract}

Keywords: Electric stimulation therapy; Muscle strength; Motor performance; Aged. 


\begin{abstract}
Resumen
Este estudio tuvo como objetivo evaluar la literatura científica de manera sistemática para analizar el efecto de la EENM en la fuerza muscular y en el desempeño motor de gerontes. La pesquisa bibliográfica fue realizada a través de publicaciones indexadas en la base de datos de la biblioteca virtual de salud, Scientific Electronic Library Online, Medical Literature Analysis and Retrieval System Online, US National Library of Medicine National Institutes of Heath e Physiotherapy evidence database. La búsqueda fue realizada utilizando los descriptores en la lengua portuguesa e inglesa: "Terapia por estimulación eléctrica", "Fuerza muscular", "Desempeño motor", "Gerontes/Ancianos". Para la organización del estudio, fueron seguidos los criterios de la revisión sistemática PRISMA (Principales ítems para relatar revisiones sistemáticas y metaanálisis). Post evaluación, se seleccionaron 5 archivos que se encuadraban en los criterios de inclusión. Diversos estudios mostraron datos que proveen evidencias de que la electroestimulación neuromuscular es un método seguro para combatir el declive muscular en ancianos, como también evita la pérdida de fuerza muscular y proporciona su incremento. Esta revisión fue conclusiva en el tocante a la eficacia de EENM, demostró que hay efectivamente aumento de la fuerza de MMII y mejora en el desempeño motor posterior a un protocolo de EENM en ancianos, mismo con gran divergencia en los parámetros y el tiempo de intervención.
\end{abstract}

Palabras clave: Terapia por estimulación eléctrica; Fuerza muscular; Desempeño motor; Anciano.

\title{
1. Introdução
}

O processo de senescência consiste em alterações fisiológicas e funcionais, resultando em incapacidades e desfechos desfavoráveis para os indivíduos idosos (Soares et al., 2017). Dentre esses desfechos está a dinapenia, a qual é definida como a perda de força muscular relacionada à idade, sendo a primeira e mais importante manifestação clínica presente nos idosos (Soares et al., 2017; Moreno et al., 2018).

Apesar da sarcopenia e da dinapenia estarem relacionadas com a idade e evoluírem muitas vezes em conjunto, Clark e Manini (2008) sugerem que esses fenômenos sejam estudados separadamente. O termo dinapenia deve então, ser utilizado para caracterizar a redução de força muscular relacionada à idade, dissociando-se do conceito de redução de massa muscular (sarcopenia).

A dinapenia impacta negativamente na vida dos idosos e reflete em significância clínica por estar associada a doenças crônicas, incapacidades, deficiências e perda de função (Borges et al., 2020). Dentre os diversos impactos da dinapenia destaca-se o declínio no desempenho motor, este envolve habilidades motoras e cognitivas para manter independência nas atividades de vida diária (AVDs), podendo gerar como consequências limitações funcionais para caminhar, levantar-se, vestirse e manter o equilíbrio. Neste mesmo cenário, piores resultados na força muscular geram consequências neuromusculares afirmadas por sinais de lentidão, fraqueza muscular e fadiga, comprometendo a independência e autonomia desses indivíduos (Santos \& Reis, 2016; Nascimento et al., 2015).

Diante do supracitado, entende-se a necessidade de desenvolver estratégias eficazes para combater os riscos da dinapenia. Dentre os recursos de fortalecimento muscular, o treinamento convencional nem sempre é acessível para essa população pelo maior custo, diminuição de mobilidade da maioria dos idosos, doenças cardiovasculares e patologias neuromusculares, o que torna o treinamento convencional nem sempre viável (Langeard et al., 2017).

Desse modo, a estimulação elétrica neuromuscular (EENM), torna-se uma alternativa possível para a prevenção da redução da força muscular e suas consequências, consistindo na aplicação de corrente elétrica sobre a superfície dos músculos a fim de promover contrações musculares com o intuito de reeducação muscular, manutenção e/ou aumento de força e resistência (Langeard et al., 2017).

O treinamento com estimulação elétrica neuromuscular pode ser tão eficiente quanto o treinamento convencional, tendo vantagens sobre o recrutamento muscular que ocorre preferencialmente de fibras musculares do tipo II, as quais são as mais afetadas no processo de envelhecimento e as responsáveis pela redução da força muscular e declínio no desempenho motor (Sousa, 2016). 
Frente ao exposto, este estudo tem como objetivo analisar os efeitos da estimulação elétrica neuromuscular na força muscular e no desempenho motor de idosos, através de uma revisão sistemática da literatura.

\section{Metodologia}

Trata-se de um estudo de revisão sistemática sem metanálise, seguindo os critérios de recomendações PRISMA (Principais Itens para Relatar Revisões Sistemáticas e Metanálises) (Moher et al., 2009). O estudo foi de caráter bibliográfico, com o objetivo de sistematizar ensaios clínicos controlados e randomizados que atendam aos critérios de inclusão definidos para a pesquisa.

Por meio da revisão sistemática - um método que permite sintetizar evidências - é possível verificar de forma crítica e interpretativa as pesquisas mais relevantes que tratam de uma temática específica, área de conhecimento ou eventos de interesse. Ele permite "identificar, selecionar e avaliar a qualidade de evidências", pois se trata de um método claro e sistemático, logo, se tratam de um tipo de estudo elaborado por uma metodologia confiável, rigorosa e possível de ser auditada. (Brasil, 2012).

Foram utilizadas as seguintes bases bibliográficas para a pesquisa: Biblioteca Virtual de Saúde (BVS), Scientific Electronic Library Online (SCIELO), Medical Literature Analysis and Retrieval System Online (MEDLINE), US National Library of Medicine National Institutesof Heath (PUBMED) e Physiotherapy evidence database (PEDro).

A busca foi realizada utilizando os descritores na língua portuguesa e inglesa: "Terapia por estimulação elétrica", "Força muscular", "Desempenho Motor", "Idoso". Os estudos foram selecionados e avaliados de forma independente por dois revisores, sendo retiradas as duplicatas. No momento de busca houve as seguintes combinações com o indicador booleano "and": "terapia por estimulação elétrica and força muscular and idoso", "terapia por estimulação elétrica and desempenho motor and idoso".

Foram incluídos estudos publicados entre os anos de 2011 a 2021 que estivessem disponíveis na íntegra de forma gratuita na língua portuguesa e inglesa e que a população alvo eram humanos. A seleção dos artigos foi realizada nas etapas: título do estudo, resumo e texto completo. Foram excluídos resumos, revisões sistemáticas e relato de casos, bem como estudos que utilizaram modelos animais.

De forma independente, os dois revisores selecionaram os estudos de acordo com as etapas descritas acima e após a seleção foram excluídas possiveis duplicatas e avaliada a qualidade metodológica dos estudos selecionados através da escala de PEDro. Essa escala avalia os seguintes quesitos: 1) critérios de eligibilidade; 2) distribuição aleatória; 3) distribuição cega; 4) diferença entre os grupos no baseliner; 5) participação cega; 6) intervenção cega; 7) avaliação cega; 8) resultados com mais de $85 \%$ da amostra; 9) situação controle; 10) resultados intergrupos; 11) medidas de precisão. O escore da escala varia de 0 a 10 pontos. Equivale a um ponto cada um dos 11 critérios se satisfeito, não sendo pontuando o primeiro item. Os estudos randomizados considerados de boa qualidade e que foram selecionados para a presente pesquisa obtiveram pontuação acima de 06 pontos na escala PEDro.

Foram encontrados nas bases de dados padronizadas durante a pesquisa, 434 artigos com os descritores definidos no início da pesquisa. Excluiu-se um total de 429 estudos, (97) por apresentar duplicação na coleta, (281) após análise do título, por não se enquadrarem ao tema proposto, (5) não estavam liberados na íntegra, (12) excluídos após leitura dos resumos, (15) a população não eram idosos, (16) utilizaram outro recurso terapêutico de estimulação elétrica, (3) não obtiveram a pontuação pré-estabelecida na escala PEDro. Dessa forma, foram selecionados 05 artigos os quais se adequaram aos critérios propostos para esta revisão sistemática. Os artigos selecionados foram organizados em um fluxograma que permitiu a sintetização das informações (Figura 1). 
Research, Society and Development, v. 10, n. 16, e90101623398, 2021

(CC BY 4.0) | ISSN 2525-3409 | DOI: http://dx.doi.org/10.33448/rsd-v10i16.23398

Figura 1. Delimitação da pesquisa e os resultados encontrados.

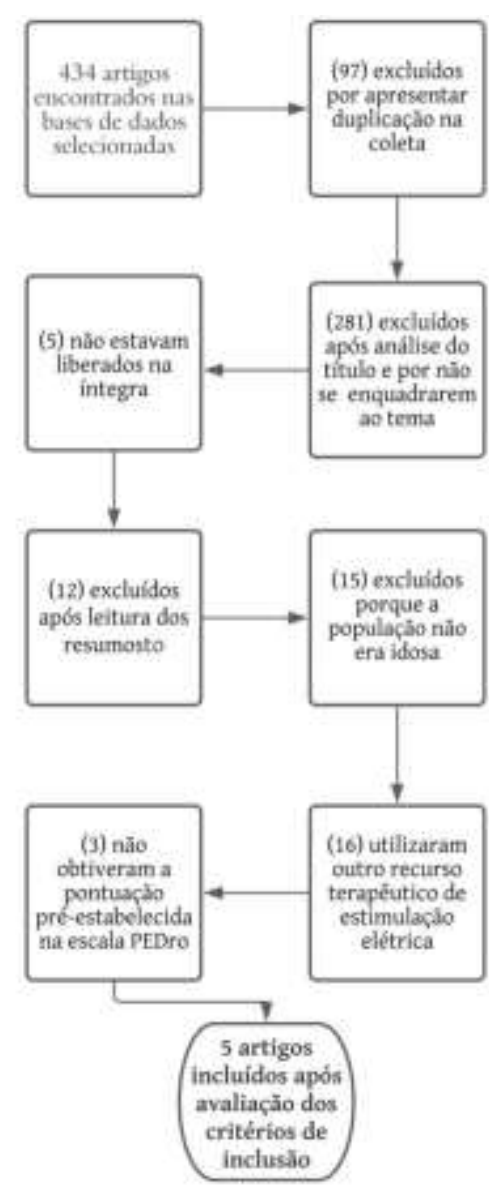

Fonte: Autores (2021).

\section{Resultados}

No Quadro 1, estão expostos os 5 artigos selecionados, organizados por autor, ano de publicação, títulos dos seus respectivos artigos, objetivo do estudo, população, os protocolos utilizados, os parâmetros da EENM e os seus principais achados. Os resultados encontrados foram tabulados e encontram-se dispostos no Quadro 1, a seguir: 
Quadro 1 - Distribuição dos artigos de acordo com os critérios metodológicos. Vitória da Conquista-BA.

\begin{tabular}{|c|c|c|c|c|c|}
\hline $\begin{array}{c}\text { Autor/ano da } \\
\text { publicação/título do } \\
\text { estudo }\end{array}$ & $\begin{array}{l}\text { Objetivo do } \\
\text { estudo }\end{array}$ & $\begin{array}{c}\text { População/amostra } \\
\text { da pesquisa }\end{array}$ & Protocolos utilizados & $\begin{array}{l}\text { Parâmetros utilizados da } \\
\text { EENM }\end{array}$ & $\begin{array}{c}\text { Principais resultados do } \\
\text { estudo }\end{array}$ \\
\hline $\begin{array}{c}\text { JANG \& PARK, } 2021 \\
\text { EFFECTS OF } \\
\text { NEUROMUSCULAR } \\
\text { ELECTRICAL } \\
\text { STIMULATION } \\
\text { COMBINED WITH } \\
\text { EXERCISES } \\
\text { VERSUS AN } \\
\text { EXERCISE } \\
\text { PROGRAM ON THE } \\
\text { PHYSICAL } \\
\text { CHARACTERISTICS } \\
\text { AND FUNCTIONS } \\
\text { OF THE ELDERLY: } \\
\text { A RANDOMIZED } \\
\text { CONTROLLED } \\
\text { TRIAL. }\end{array}$ & $\begin{array}{l}\text { Avaliar a adição } \\
\text { da EENM aos } \\
\text { exercícios de } \\
\text { fortalecimento de } \\
\text { membros } \\
\text { inferiores para } \\
\text { prevenção de } \\
\text { quedas em idosos } \\
\text { por um período } \\
\text { relativamente } \\
\text { curto de } 4 \\
\text { semanas. }\end{array}$ & $\begin{array}{c}\text { Ensaio clínico } \\
\text { randomizado com } 30 \\
\text { mulheres idosas com } \\
65 \text { anos ou mais. } \\
\text { Foram categorizadas } \\
\text { aleatoriamente em três } \\
\text { grupos: grupo controle } \\
\text { (CON, } n=8), \text { grupo de } \\
\text { exercícios }(\mathrm{EX}, n=10) \\
\text { e grupo de EENM com } \\
\text { exercícios }(\mathrm{EX}+ \\
\text { NMES, } n=9) .\end{array}$ & $\begin{array}{l}\text { A intervenção de exercícios } \\
\text { foi realizada } 3 \text { vezes por } \\
\text { semana, durante } 4 \text { semanas. } \\
\text { Foi dividida em } 3 \text { partes: } \\
\text { alongamento dos músculos de } \\
\text { MMSS e } \\
\text { MMII; fortalecimento de } \\
\text { MMII incluíram elevação da } \\
\text { perna reta e ponte em posição } \\
\text { supina, abdução lateral do } \\
\text { quadril, abdução e extensão } \\
\text { do quadril em pé, e elevação } \\
\text { do calcanhar e agachamento } \\
\text { em pé. Além disso, na } \\
\text { posição sentada, os sujeitos } \\
\text { realizaram exercícios de } \\
\text { extensão de joelho, adução de } \\
\text { quadril e sentar para levantar. } \\
\text { Além disso, o grupo EENM + } \\
\text { exercício utilizou } 10 \text { min de } \\
\text { aquecimento, } 40 \text { min de } \\
\text { exercícios de fortalecimento } \\
\text { de MMII e } 10 \text { min de } \\
\text { resfriamento. }\end{array}$ & $\begin{array}{l}\text { Os eletrodos foram fixados } \\
\text { nos músculos vasto medial e } \\
\text { vasto lateral com os } \\
\text { seguintes parâmetros: } \\
\text { frequência de } 35 \mathrm{~Hz} \text { e uma } \\
\text { duração de } 300 \mu \mathrm{s} \text {, definida } \\
\text { como uma onda quadrada } \\
\text { por } 20 \text { min para provocar a } \\
\text { contração muscular. Uma } \\
\text { corrente de pulso (mA) foi } \\
\text { aplicada para provocar } \\
\text { contrações musculares sem } \\
\text { dor ou desconforto (média } \\
\text { de } 10-12 \mathrm{~mA} \text { ) }\end{array}$ & $\begin{array}{l}\text { As comparações entre os } \\
\text { três grupos mostraram que } \\
\text { o TUG diminuiu } \\
\text { significativamente e o teste } \\
\text { de equilíbrio em Y } \\
\text { aumentou } \\
\text { significativamente no } \\
\text { grupo EENM + exercício } \\
(p<0,05) \text {. } \\
\text { Não houve diferença } \\
\text { significativa na massa } \\
\text { muscular esquelética, } \\
\text { massa de gordura corporal, } \\
\text { circunferência da } \\
\text { panturrilha e força de } \\
\text { preensão manual entre } \\
\text { cada grupo (p > } 0,05)\end{array}$ \\
\hline $\begin{array}{l}\text { CANDAN et al., } 2019 . \\
\text { Effects of } \\
\text { neuromuscular } \\
\text { electrical stimulation } \\
\text { of quadriceps on the } \\
\text { quadriceps strength } \\
\text { and functional } \\
\text { performance in } \\
\text { nursing home } \\
\text { residents: A } \\
\text { comparison of short } \\
\text { and long stimulation } \\
\text { periods. }\end{array}$ & $\begin{array}{l}\text { Determinar o } \\
\text { efeito de dois } \\
\text { períodos de } \\
\text { estimulação } \\
\text { elétrica } \\
\text { neuromuscular } \\
\text { (EENM) na força } \\
\text { do quadríceps } \\
\text { (QS) e no } \\
\text { desempenho } \\
\text { funcional em } \\
\text { residentes de } \\
\text { asilos. }\end{array}$ & $\begin{array}{l}\text { Trata-se de um ensaio } \\
\text { clínico simples-cego } \\
\text { (avaliadores) com } 44 \\
\text { idosos (idade } \geq 65 \\
\text { anos). Os participantes } \\
\text { foram divididos } \\
\text { aleatoriamente em } \\
\text { dois grupos - o grupo } \\
\text { de curto período de } \\
\text { estimulação (SNMES } \\
\text { n=22) e grupo longo } \\
\text { período de estimulação } \\
\text { (LNMES n=22) }\end{array}$ & $\begin{array}{c}\text { O participante estava na } \\
\text { posição sentada durante a } \\
\text { estimulação } \\
\text { e os joelhos foram } \\
\text { semiflexionados aos } 30^{\circ} \text {. O } \\
\text { período de estimulação } \\
\text { foi de } 20 \text { minutos no total } \\
\text { para cada grupo. No entanto, } \\
\text { a corrente foi entregue em } \\
\text { conjuntos, que diferiram entre } \\
\text { os grupos. A corrente foi } \\
\text { aplicada por } 5 \text { min em quatro } \\
\text { séries para o grupo SNMES, } \\
\text { e por } 10 \text { min em duas séries } \\
\text { para o grupo LNMES. Um } \\
\text { descanso de } 10 \text { minutos } \\
\text { período foi deixado entre as } \\
\text { séries em cada grupo. }\end{array}$ & $\begin{array}{c}\text { Quatro eletrodos de } \\
\text { superfície autoadesivos } \\
(5 \times 5 \mathrm{~cm}) \text { foram colocados } \\
\text { nos pontos motores do vasto } \\
\text { medial } \\
\text { e reto femoral. Com os } \\
\text { seguintes parâmetros: onda } \\
\text { simétrica, bifásica e } \\
\text { quadrada com uma } \\
\text { frequência de } \\
100 \text { Hz e uma duração de } \\
\text { pulso de } 400 \mu \text { s. A } \\
\text { intensidade da corrente foi } \\
\text { aumentada até que a } \\
\text { contração visível das fibras } \\
\text { musculares fosse } \\
\text { Observada.ON: } 5 \mathrm{~s} \text { OFF: } 15 \mathrm{~s}\end{array}$ & $\begin{array}{l}\text { A força do quadríceps } \\
\text { isométrico não mudou } \\
\text { dentro dos grupos ou entre } \\
\text { os grupos. Curiosamente, a } \\
\text { pontuação teste de pé ne } \\
\text { cadeira de } 30 \text { segundos } \\
\text { (30CST) aumentou para } \\
\text { ambos os grupos, mas as } \\
\text { melhorias foram } \\
\text { semelhantes entre os } \\
\text { grupos. A pontuação da } \\
\text { escala de equilíbrio de } \\
\text { Berg, o tempo do teste } \\
\text { TUG e a distância do teste } \\
\text { de caminhada de } 6 \text { minutos } \\
\text { também melhoraram em } \\
\text { ambos os grupos. No } \\
\text { entanto, nenhuma } \\
\text { diferença em qualquer uma } \\
\text { das medidas de resultados } \\
\text { funcionais foi observada } \\
\text { entre os grupos. }\end{array}$ \\
\hline $\begin{array}{l}\text { ZINGLERSEN et al., } \\
2018 \\
\text { Can electrical } \\
\text { stimulation enhance } \\
\text { effects of a functional } \\
\text { training program in } \\
\text { hospitalized geriatric } \\
\text { patients? }\end{array}$ & $\begin{array}{l}\text { Investigar as } \\
\text { mudanças na } \\
\text { função e } \\
\text { desempenho em } \\
\text { pacientes } \\
\text { geriátricos } \\
\text { hospitalizados } \\
\text { realizando um } \\
\text { programa de } \\
\text { exercícios } \\
\text { funcionais diários } \\
\text { com e sem EENM } \\
\text { simultânea para } \\
\text { os músculos } \\
\text { extensores do } \\
\text { joelho. }\end{array}$ & $\begin{array}{l}\text { Trata-se de um ensaio } \\
\text { quase randomizado } \\
\text { controlado em } \\
\text { pacientes geriátricos } \\
\text { hospitalizados ( } \mathrm{N}= \\
16) \text {. Exercícios } \\
\text { funcionais diários + } \\
\text { EENM (FT + EENM } \\
\mathrm{N}=8) ;(\mathrm{FT} \mathrm{N}=8) .\end{array}$ & $\begin{array}{c}\text { Os pacientes realizaram } \\
\text { diariamente um programa de } \\
\text { exercícios funcionais simples } \\
\text { com base na cadeira em pé, } \\
\text { com ou sem EENM } \\
\text { simultânea para os músculos } \\
\text { extensores do joelho . A } \\
\text { função física foi avaliada no } \\
\text { dia } 2 \text { e 6-10 da hospitalização } \\
\text { com o Índice de Mobilidade } \\
\text { De Morton (DEMMI), um } \\
\text { teste de suporte de cadeira de } \\
\text { 30s (30s CST) e um teste de } \\
\text { velocidade de marcha de } 4 \\
\text { metros (4 m-GST) O } \\
\text { treinamento foi comparados a } \\
\text { um grupo de controle } \\
\text { histórico semelhante (CON, N } \\
=48 \text { ) recebendo apenas o } \\
\text { tratamento padrão. }\end{array}$ & $\begin{array}{c}\text { Um eletrodo autoadesivo (10 } \\
\text { x } 5 \mathrm{~cm} \text { ) foi colocado na } \\
\text { linha média de ambas as } \\
\text { coxas } \\
5 \mathrm{~cm} \text { distal à crista inguinal } \\
\text { e um eletrodo menor ( } 5 \text { x } 5 \\
\mathrm{~cm} \text { ) foi colocado na parte } \\
\text { distal de ambos os músculos } \\
\text { vasto lateral e vasto } \\
\text { Medial (VL e VM) com os } \\
\text { parâmetros: Intensidade } \\
\text { máxima de estimulação= } \\
120 \text { mA, F= 35 Hz, Dp= } 400 \\
\mu \mathrm{s} ; \text { ON/OFF=5/10s } \\
\text { (familiarização), } 5 / 5 \mathrm{~s} \text { ou } 2,5 \\
/ 2,5 \mathrm{~s} \\
\text { com uma fase de } \\
\text { aceleração/desaceleração de } \\
0,5 \mathrm{~s} \text {. }\end{array}$ & $\begin{array}{l}\text { Durante os } 6-10 \text { dias de } \\
\text { hospitalização, ambos os } \\
\text { grupos melhoraram em } \\
\text { todas as medidas } \\
\text { funcionais ( } \mathrm{p}<0,05) \text {, mas } \\
\text { sem diferença entre os } \\
\text { grupos ( } \mathrm{p}>0,05) \text {. } \\
\text { Comparado ao tratamento } \\
\text { padrão, o treinamento } \\
\text { resultou em melhorias } \\
\text { significativamente maiores } \\
\text { no } 30 \text { s-CST } \\
\text { (TREINAMENTO: }+3,8 \\
\text { repetições; CON: }+1,4 \\
\text { repetições, } p<0,01 \text { ), mas } \\
\text { não no teste DEMMI e no } \\
4 \text { m-GST. }\end{array}$ \\
\hline
\end{tabular}




\begin{tabular}{|c|c|c|c|c|c|}
\hline $\begin{array}{l}\text { KARLSEN et al., } \\
2020 \\
\text { Neuromuscular } \\
\text { Electrical Stimulation } \\
\text { Preserves Leg Lean } \\
\text { Mass in Geriatric } \\
\text { Patients. }\end{array}$ & $\begin{array}{c}\text { Examinar as } \\
\text { alterações na } \\
\text { massa magra } \\
\text { durante a } \\
\text { hospitalização em } \\
\text { pacientes } \\
\text { geriátricos e o } \\
\text { efeito da ativação } \\
\text { muscular por } \\
\text { estimulação } \\
\text { elétrica } \\
\text { neuromuscular. }\end{array}$ & $\begin{array}{l}\text { Treze pacientes (69-94 } \\
\text { anos) em uma } \\
\text { enfermaria geriátrica } \\
\text { completaram os testes } \\
\text { na admissão ao } \\
\text { hospital (dias 2-3) e na } \\
\text { alta (dias 8-10). }\end{array}$ & $\begin{array}{l}\text { A intervenção consistiu em } \\
\text { uma sessão diária de E-Stim } \\
\text { do músculo vasto lateral (VL) } \\
\text { e vasto medial (VM) de uma } \\
\text { perna, enquanto a outra perna } \\
\text { serviu como uma perna de } \\
\text { controle (CON). O E-Stim foi } \\
\text { atribuído aleatoriamente à } \\
\text { perna mais forte ou mais } \\
\text { fraca, começando com a } \\
\text { familiarização com o } \\
\text { protocolo. }\end{array}$ & $\begin{array}{c}\text { Um eletrodo autoadesivo de } \\
10 \times 5 \mathrm{~cm} \text { foi colocado na } \\
\text { linha média da parte anterior } \\
\text { da coxa, } 5 \mathrm{~cm} \text { distal à prega } \\
\text { inguinal. Um eletrodo de } 5 \times \\
5 \mathrm{~cm} \text { foi colocado na parte } \\
\text { distal do VL e VM, } \\
\text { respectivamente. } \\
\text { Resumidamente, o protocolo } \\
\text { consistia em uma sequência } \\
\text { de aquecimento / } \\
\text { resfriamento de } 5 \text { min }(5 \mathrm{~Hz}, \\
250 \mu \mathrm{s}) \text { e uma sequência de } \\
\text { estimulação de } 30 \text { min }(5 \mathrm{~s} \\
\text { ON / } 10 \mathrm{~s} \text { OFF, } 100 \mathrm{~Hz}, 400 \\
\mu \mathrm{s}, 0 \text { - } 120 \mathrm{~mA}) \text { com uma } \\
\text { subida/descida de } 0,75 \mathrm{~s} \mathrm{e} \\
\text { uma fase de contração de } \\
3,5 \mathrm{~s}\end{array}$ & $\begin{array}{l}\text { Não houve declínio nas } \\
\text { pontuações dos testes } \\
\text { funcionais de corpo inteiro, } \\
\text { mas deve-se observar que } \\
\text { esses testes foram } \\
\text { incluídos para fins } \\
\text { descritivos gerais, embora } \\
\text { não sejam adequados para } \\
\text { avaliação de uma } \\
\text { intervenção unilateral. No } \\
\text { entanto, em comparação } \\
\text { com a perna CON, houve } \\
\text { mudanças gerais favoráveis } \\
\text { na expressão do } \\
\text { gene associado à atrofia } \\
\text { das fibras musculares } \\
\text { (miostatina, MURF1, } \\
\text { MAFbx), remodelação da } \\
\text { matriz extracelular } \\
\text { (Colágeno 1, TenascinaC) } \\
\text { e marcadores de atividade } \\
\text { celular (CD68, Ki67), } \\
\text { juntos demonstrando uma } \\
\text { resposta positiva no nível } \\
\text { celular. }\end{array}$ \\
\hline $\begin{array}{l}\text { RAK et al., } 2017 \\
\text { Short-term } \\
\text { microcurrent electrical } \\
\text { neuromuscular } \\
\text { stimulation to improve } \\
\text { muscle function in the } \\
\text { elderly: A randomized, } \\
\text { double-blinded, sham- } \\
\text { controlled clinical trial }\end{array}$ & $\begin{array}{l}\text { Avaliar os efeitos } \\
\text { da EENM de } \\
\text { curto prazo } \\
\text { na força, } \\
\text { resistência e } \\
\text { atividade } \\
\text { muscular em } \\
\text { idosos. }\end{array}$ & $\begin{array}{c}38 \text { participantes idosos } \\
\text { saudáveis com } 65 \text { anos } \\
\text { ou mais foram inscritos } \\
\text { e divididos } \\
\text { aleatoriamente em } 2 \\
\text { grupos de estimulação: } \\
\text { grupo EENM real } \\
(\mathrm{N}=18) \text { ou simulado } \\
(\mathrm{N}=18) .\end{array}$ & $\begin{array}{l}\text { Foram aplicados aos } 8 \text { pontos } \\
\text { anatômicos do braço e perna } \\
\text { dominantes durante o curso } \\
\text { de } 40 \text { minutos. Foram } \\
\text { realizados testes de função } \\
\text { muscular no início e após a } \\
\text { intervenção. }\end{array}$ & $\begin{array}{c}\text { Foram aplicados } 2 \\
\text { dispositivos de } \\
\text { microcorrentes idênticos em } \\
\text { cada membro superior e } \\
\text { inferior. } \\
\text { A frequência era de } 8 \mathrm{~Hz} \text { e a } \\
\text { intensidade de } 25 \mu \mathrm{A}\end{array}$ & $\begin{array}{l}\text { No grupo EENM real, os } \\
\text { valores médios da FPM e o } \\
\text { número de flexões } \\
\text { plantares aumentaram } \\
\text { significativamente, mas o } \\
\text { valor RMS do sinal } \\
\text { eletromiográfico diminuiu } \\
\text { significativamente após a } \\
\text { intervenção real } \\
(P<0,05) \text {. No entanto, o } \\
\text { grupo simulado EENM } \\
\text { mostrou uma diminuição } \\
\text { significativa no número de } \\
\text { flexões plantares e no } \\
\text { tempo total para TRH após } \\
\text { a estimulação simulada } \\
\text { (P < } 0,05) \text {. }\end{array}$ \\
\hline
\end{tabular}

Fonte: Bases de dados Biblioteca Virtual de Saúde (BVS), Medical Literature Analysis and Retrieval System Online (MEDLINE), US National Library of Medicine National Institutesof Heath (PUBMED) e Physiotherapy evidence database (PEDro).

\section{Discussão}

Os principais achados da maioria dos estudos demonstraram aumento da força de MMII e melhora no desempenho motor após um protocolo de EENM em idosos, mesmo com grande divergência nos parâmetros e no tempo de intervenção. Ao avaliar os resultados do ponto de vista funcional, todos os estudos melhoraram o desempenho funcional, mas nem todos mostraram diferenças significativas entre grupos.

As formas de avaliação da força muscular se deram através de dinamômetro digital e dinamômetro manual hidráulico para indicar a força geral do corpo e função física. A avaliação do desempenho funcional foi realizada através de testes como Sit-to-Stand cinco vezes; Timed Up-and-Go; teste de apoio de uma perna; teste de equilíbrio em Y; teste de caminhada de 6 minutos (TC6); teste de pé de cadeira de 30 segundos (30CST) e escala de equilíbrio de Berg. Tais instrumentos são de grande importância visto que, apresentam validade, fácil reprodutibilidade e aplicabilidade sendo fundamentais para avaliar força/resistência de membros inferiores, flexibilidade e força global (Trindade, 2014).

O fortalecimento muscular parece ser concomitante no aumento da síntese proteica, bem como também à adaptação neural e melhor coordenação (Macaluso \& De Vito, 2004; Zampieri et al., 2014). Porém, o exercício físico tradicional para o fortalecimento muscular nem sempre é uma opção para indivíduos mais velhos. Condições patológicas e o processo natural do envelhecimento limitam a eficácia do exercício físico. Nesse contexto, a EENM pode ser uma alternativa, no estudo de Krenn 
et al. (2011), por exemplo, a EENM induziu a regulação positiva de marcadores relevantes das células satélites diferenciadoras e do remodelamento da matriz extracelular, mantendo a função das células e reduzindo assim a fibrose.

A EENM se mostra uma alternativa eficaz para preservar/recuperar a função muscular, visto que estimula diretamente a síntese de proteínas do músculo esquelético (Strasser et al., 2009; Wall et al., 2012). Além disso, a EENM tem a vantagem de ser capaz de preferencialmente atingir fibras musculares do tipo II, fibras estas mais afetadas no envelhecimento e responsáveis pelo declínio no desempenho motor e na força muscular (Pfeifer et al., 2002).

Na contração muscular voluntária, primariamente são contraídas as fibras de contração lenta (tipo I), já na estimulação elétrica neuromuscular ocorre que as fibras de contração rápida (tipo II) são recrutadas primariamente. A intensidade da corrente é o que determina a contração muscular e consequentemente a produção de mais força (Montenegro et at., 2019).

Candan et al. (2019), avaliou a força do quadríceps e o desempenho funcional de idosos com o intuito de determinar se existe diferença no efeito de dois períodos de EENM - curto período de estimulação e longo período de estimulação-. No entanto, nenhuma diferença em qualquer uma das medidas de resultados funcionais foi observada entre os grupos. Sendo assim, dois períodos de EENM tiverem respostas funcionais similares. Porém, no que tange a força muscular do quadríceps, não houve aumento significativo.

Destaca-se que evidências sugerem que a intensidade deve estar em um nível máximo tolerável para atingir o efeito de fortalecimento (Kern et al., 2014). O aprendizado motor e a facilitação neural gera um recrutamento mais eficiente das unidades motoras com o maior impulso e maior frequência que são disparados (Chaves, 2020). No entanto, no estudo de Candan et al (2019) a intensidade foi mínima até a contração visível, o que pode ter responsabilidade sobre o estudo a respeito do ganho de força muscular.

Visto que a intervenção com EENM parece tão eficaz quanto treinamento voluntário, pode ser interessante para população idosa, mesmo não tendo sido apresentado uma superioridade neste estudo. Pois desencadeia miofibrilas rápidas e lentas, porém mais estudos precisam ser realizados a fim de determinar se a EENM deve ser a escolha primária para ganho de força muscular e melhora no desempenho funcional em idosos. Em um estudo de Caggiano et al (1994), foi realizada a comparação entre os efeitos de EENM e o nível de atividade dos participantes, sendo que o treinamento se mostrou mais eficiente em pessoas que têm menor atividade (aumento de 12\%) do que para os idosos mais ativos (aumento de 5\%).

Em virtude disso, a estimulação elétrica neuromuscular parece ser uma interessante alternativa de treinamento muscular, adequada e eficiente para idosos que são menos ativos e não são capazes de realizarem exercícios tradicionais.

Os estudos abordados na revisão mostraram respostas significativas no desempenho motor e força muscular gerada pela EENM em idosos, mas não houve melhora significativa em comparação entre grupos que realizaram apenas exercícios tradicionais. Sendo assim, há evidências que favorecem a utilização da EENM em idosos, com resultados semelhantes aos encontrados pelos exercícios ativos. Destacamos, porém, a necessidade de uma maior gama de estudos sobre a temática, bem como a dificuldade de estabelecer protocolos mais eficazes.

\section{Conclusão}

A análise da literatura evidenciou efeitos positivos no uso da estimulação elétrica neuromuscular no ganho de força muscular e no desempenho motor de idosos. Dessa forma, recomenda-se a EENM nas práticas clínicas de reabilitação e prevenção em indivíduos idosos, sendo uma terapia segura e eficiente de reabilitação neuromuscular, melhorando a qualidade de vida desta população como também, proporcionando maior segurança a esse público, afinal, o ganho de força muscular e um melhor desempenho motor podem prevenir quedas, fraturas e hospitalizações.

No entanto, recomenda-se a realização de novos estudos que busquem identificar as possíveis relações desta intervenção com os exercícios tradicionais, qual a sua melhor forma de ser administrada, sendo isolada ou combinada, bem 
como se existem diferenças significativas em relação a outros tipos de condutas para ganho de força muscular e desempenho motor que seja confortável e de fácil realização para idosos. Sugere-se a realização de mais estudos voltados a essa temática com o objetivo de compreender e avaliar quais protocolos teriam melhores benefícios, como também, aconselha-se novos estudos nos quais sejam investigados os resultados do impacto da terapia por estimulação elétrica no equilíbrio em idosos.

\section{Referências}

Alexandre, T. D. S., Duarte, Y. A. D. O., Santos, J. L. F., \& Lebrão, M. L. (2019). Prevalence and associated factors of sarcopenia, dynapenia, and sarcodynapenia in community-dwelling elderly in São Paulo-SABE Study. Revista Brasileira de Epidemiologia, 21.

Baptista, R. R., \& Vaz, M. A. (2009). Arquitetura muscular e envelhecimento: adaptação funcional e aspectos clínicos; revisão da literatura. Fisioterapia e Pesquisa, 16, 368-373.

Bessa, L. B. R. S., \& Barros, N. V. (2009). Impacto da sarcopenia na funcionalidade de idosos [monografia]. Belo Horizonte: Escola de Educação Física, Fisioterapia e Terapia Ocupacional, Universidade Federal de Minas Gerais.

Borges, V. S., Lima-Costa, M. F. F., \& Andrade, F. B. D. (2020). A nationwide study on prevalence and factors associated with dynapenia in older adults: ELSI-Brazil. Cadernos de saude publica, 36, e00107319.

Brandão, W. G., Lisboa, R. B., dos Santos Barros, E., Oliveira-Silva, I., \& Pereira, L. C. (2020). Efeitos da eletroestimulação neuromuscular sobreposta ao treinamento de força sobre os níveis de lactato sanguíneo. International Journal of Movement Science and Rehabilitation, 2(1), 45-54.

Brasil. Ministério da Saúde. Secretaria de Ciência, Tecnologia e Insumos Estratégicos. Departamento de Ciência e Tecnologia. Diretrizes metodológicas: elaboração de revisão sistemática e metanálise de ensaios clínicos randomizados/ Ministério da Saúde, Secretaria de Ciência, Tecnologia e Insumos Estratégicos, Departamento de Ciência e Tecnologia. - Brasília: Editora do Ministério da Saúde, 2012.

Caggiano, E., Emrey, T., Shirley, S., \& Craik, R. L. (1994). Effects of electrical stimulation or voluntary contraction for strengthening the quadriceps femoris muscles in an aged male population. Journal of Orthopaedic \& Sports Physical Therapy, 20(1), 22-28.

Constantino, A., Rocha, E. S., de Oliveira, O. M. P., \& Monteiro, M. M. D. O. (2019). Declínios fisiológicos e fisiopatológicos do sistema locomotor durante o envelhecimento humano: uma revisão bibliográfica. Anais VI CIEH, Campina Grande: Realize Editora, 1-8.

C Clark, B., \& L Taylor, J. (2011). Age-related changes in motor cortical properties and voluntary activation of skeletal muscle. Current aging science, 4(3), 192-199.

Clark, B. C., \& Manini, T. M. (2008). Sarcopeniał dynapenia. The Journals of Gerontology Series A: Biological Sciences and Medical Sciences, 63(8), 829834 .

Critchley, K., Kokubu, M., Iemitsu, M., Fujita, S., \& Isaka, T. (2014). Age-related differences in the availability of visual feedback during bimanual pinch. European journal of applied physiology, 114(9), 1925-1932.

De Carvalho, B.A. et al. (2020). Efeito independente e combinado de treinamento de resistência progressiva baseado em casa e suplementação nutricional na força muscular, massa muscular e função física em idosos dinapênicos com baixa ingestão de proteína: um ensaio clínico randomizado. Arquivos de Gerontologia e Geriatria.

Dias, R. M. R., Cyrino, E. S., Salvador, E. P., Nakamura, F. Y., Pina, F. L. C., \& Oliveira, A. R. D. (2005). Impacto de oito semanas de treinamento com pesos sobre a força muscular de homens e mulheres. Revista brasileira de medicina do esporte, 11, 224-228.

Eletroterapia: prática baseada em evidências. Manole, 2003.

Fechine, B. R. A., \& Trompieri, N. (2012). O processo de envelhecimento: as principais alterações que acontecem com o idoso com o passar dos anos. InterSciencePlace, 1(20).

Hamil, J., \& Knutzen, K. M. (2008). Bases biomecânicas do movimento humano.

Kern, H., Barberi, L., Löfler, S., Sbardella, S., Burggraf, S., Fruhmann, H., \& Musaro, A. (2014). Electrical stimulation counteracts muscle decline in seniors. Frontiers in aging neuroscience, $6,189$.

Kobayashi, K., Imagama, S., Ando, K., Nakashima, H., Machino, M., Morozumi, M., \& Hasegawa, Y. (2020). Dynapenia and physical performance in community-dwelling elderly people in Japan. Nagoya Journal of Medical Science, 82(3), 415.

Landi, F., Liperoti, R., Fusco, D., Mastropaolo, S., Quattrociocchi, D., Proia, A., \& Onder, G. (2012). Sarcopenia and mortality among older nursing home residents. Journal of the American Medical Directors Association, 13(2), 121-126.

Langeard, A., Bigot, L., Chastan, N., \& Gauthier, A. (2017). Does neuromuscular electrical stimulation training of the lower limb have functional effects on the elderly?: A systematic review. Experimental gerontology, 91, 88-98.

Macedo, T. A., de Oliveira, D. V., Domingues, W. J. R., Adriana, T., \& Martineli, P. (2018). Efeitos de um programa de exercícios resistidos na força muscular de idosos effects of an exercise program resisted in the muscle force of elderly.

Maior, A. S., \& Alves, A. (2003). A contribuição dos fatores neurais em fases iniciais do treinamento de força muscular: uma revisão bibliográfica. Motriz, 9(3), 161-8. 
Research, Society and Development, v. 10, n. 16, e90101623398, 2021

(CC BY 4.0) | ISSN 2525-3409 | DOI: http://dx.doi.org/10.33448/rsd-v10i16.23398

Mancini, R.B., et al. (2019). Prevalência e fatores associados à dinapenia em idosos institucionalizados. Um estudo transversal. Nutrição, saúde e atividade física.

Mancini, R. B., Junior, J. P. D. S., Araujo, T. L., Matsudo, S. M., \& Matsudo, V. (2019). Prevalência e fatores associados à dinapenia em idosos institucionalizados. Um estudo transversal. Diagn. tratamento, 111-118.

Mendonça, C. D. S., Moura, S. K., \& Lopes, D. T. (2018). Benefícios do treinamento de força para idosos: revisão bibliográfica. Revista Campo do Saber, 4(1).

Muniz, C. F. Revista Brasileira de Atividade Física (ISSN: 2317-1634) 2 (Doctoral dissertation, Universidade Estadual Paulista).

Nascimento, C. P., Lago, L. S., de Almeida, R. F. F., Gusmão, M. F. S., Duarte, S. F. P., \& dos Reis, L. A. (2015). Desempenho motor em idosos participantes de grupos de convivência. Revista Pesquisa em Fisioterapia, 5(2).

Novais, M. M., Araújo, C. M., Bôas, S. V., Prates, R. V., de Souza Pinto, D. D., \& dos Reis, L. A. (2016). Avaliação de indicadores de desempenho funcional de idosos longevos residentes em domicílio. Arquivos de Ciências da Saúde, 23(3), 67-72.

de Oliveira, E. N., dos Santos, K. T., \& dos Reis, L. A. (2017). Força de preensão manual como indicador de funcionalidade em idosos. Revista Pesquisa em Fisioterapia, 7(3), 384-392.

Pires, K. F. (2004). Análise dos efeitos de diferentes protocolos de eletroestimulação neuromuscular através da frequiência mediana. Rev. bras. ciênc. mov, 2528 .

Ramos, E. D. A., \& Sandoval, R. A. (2018). Análise da força muscular, flexibilidade e equilíbrio de idosos praticantes de treinamento funcional. Revista científica da Escola Estadual de Saúde Pública de Goiás" Cândido Santiago", 4(1), 046-053.

Roberto, A. E. (2006). Eletroestimulação: o exercício do futuro. In Eletroestimulação: o exercício do futuro (pp. 198-198).

Robertson, V. R. (2009). A et all. Eletroterapia Explicada. Princípios e Prática. (4a ed.).

Rossi, E. (2008). Envelhecimento do sistema osteoarticular. Einstein, S7-S12.

Santos, K. A., \& dos Reis, L. A. (2016). Fatores associados ao desempenho motor em idosos participantes de grupos de convivência. Arquivos de Ciências da Saúde, 23(3), 73-79.

Soares, A. V., Marcelino, E., Maia, K. C., \& Borges, N. G. (2017). Relação entre mobilidade funcional e dinapenia em idosos com fragilidade. Einstein (São Paulo), 15, 278-282.

Sousa, E. F. D. (2016). Efeitos da eletroestimulação neuromuscular em pacientes críticos: uma revisão de literatura. 